\title{
A Mathematical Proof Concerning the Geometrical Aspect of Very Low Index of Difficulty in Multidirectional Tapping Task of the ISO 9241 - Part 411
}

\author{
Reyna Marsya Quita ${ }^{*}$, and Romy Budhi Widodo \\ Ma Chung University, Informatics Department, Villa Puncak Tidar N-01 Malang, 65151, Indonesia
}

\begin{abstract}
Through mathematics, this paper works to analyse the Index of Difficulty in ISO9241-Part 411: Evaluation methods for the design of physical input devices. It was proven that all the target circles always intersect for very low Index of Difficulty; which conjectured to disturb the experiment or affect the focus of the subject. Some examples are provided, in addition, to support the proof. We hope this proof will contribute to the improvement of ISO9241, where the result might become a special attention to the designer of the experiment in human-computer interaction field; especially in checking the influence of overlap area in the human factor.
\end{abstract}

Key words: Fitts' law, Human-computer interaction evaluation, Index of difficulty, ISO 9241

\section{Introduction}

The existence of ISO standard helps the researchers in evaluating pointing devices. In 2012, ISO published ISO/TS 9241-Part 411 that named Ergonomics of human-system interaction: Evaluation methods for the design of physical input devices. This standard helps the researchers to adopt a uniform measurement and avoid the bias of the results; also establish procedures of testing for evaluating pointing devices created by different companies.

The value of ISO9241 standard already widely known by academic researchers. Various studies already apply this standard as a guideline for evaluating non-keyboard input devices. Norman and Norman [1] compared the characteristics of remote pointing devices, i.e. relative versus absolute pointing devices; as an evaluating criterion instrument, they used ISO 9241-9. In [2], Natapov, et al. also use this standard as a basis for comparison between Wii remote and Wii classic controller. MacKenzie, et al. [3] explained theoretical basis, computational techniques and the evaluation of four devices (mouse, trackball, joystick, and touch pad) to validate the measurement; where the tool to evaluate the performance was using ISO 9241-9. Oh, et al. [4] wanted to make an interface with multiuser using modified laser pointer and compare it with the mouse as the baseline; the

*Corresponding author: reynaquita2905@gmail.com 
performance evaluation and comparison according to ISO9241-9 standard also presented. In [5], Widodo, et al. was investigating the performance of two laser spot clicking techniques. They also used ISO/TS 9241-411 procedure as a quantitate performance test based on Fitts' one-direction tapping test. Generally speaking, all of these previous papers used ISO 9241 as a basis for evaluating the performance of pointing devices. Evidently, based on these works, ISO has become the attention of the researchers; which make the importance of ISO as the measuring standard is undeniable.

ISO suggested a variety of range of difficulties to test the input devices, which called Index of Difficulty (ID). The ranges of difficulties are high, medium, low, and very low. A problem emerges when a very low range is applied in circular order for multi-directional tapping task; the issue is the appearance of intersection area between targets circles. By cause of this overlapping, it seems ambiguous which next target to be reached; it is surmised that the intersection affects the focus of the subject in tapping test. It implies the effectiveness and efficiency of this paradigm are vague. This intersection develops after some cases had been attempted for very low ID; however, some cases cannot assert as general. To make it general, mathematics is required to prove and analyse the case.

Therefore, the purpose of this paper is to prove and analyse mathematically that all the target circles always intersect for very low ID; which presumed to disturb the experiment or affect the subject's focus. To support the proof, some examples also given. We hope this proof will give a contribution to the enhancement of ISO9241, and the reassessment to find the new solution will be done for a very low index of difficulty classification.

For readers' benefit, a summary of ISO/TS 9241-411 is presented in the following section. Followed by mathematical proof and examples to assist the proof. And lastly, there is a conclusion that can be embraced by researchers.

\section{Evaluation of very low index of difficulty}

\subsection{ISO/TS 9241-411}

The relationship between controls and displays in human-factor performance is evaluated in the interface [6]. The evaluation procedures to measure interface's performance using experimental protocol are depicted in ISO 9241. The ISO is composed of nine series, 100 to 900 series. The only series that discusses physical input data is series 400 . The method to evaluate the design of physical input data is contained in series 400 part 411 . A procedure of evaluation is described in this standard to measure user performance for assessing the efficiency and effectiveness of all input devices.

Performance is measured by task performances on any of these tasks: selecting, pointing, dragging, free-hand input, and tracing. The performances of ISO standard are presented as one-direction tapping task, multi-directional tapping task, dragging task, and tracing task. It is not compulsory to test all of these performances to the input devices; it is decided by the expected use of the device [7].

The main ISO dependent measure is Throughput (TP). TP is the rate of information transfer when an input device is operated by a user to control a pointer on a display. The unit of TP is bits per second (bps), and it merges speed and accuracy of a device in responses.

$$
\begin{aligned}
& T P=\frac{I D_{e}}{T M} \\
& I D_{e}=\log _{2}\left(\frac{D+w_{e}}{w_{e}}\right)
\end{aligned}
$$




$$
w_{e}=4.133 \cdot s_{x}
$$

TP is calculated by dividing the effective Index of Difficulty ( $I D_{e}$, in bits, measures user accuracy required in a test) by the average movement time (TM, in seconds, is calculated from the first movement of input devices to the target). The effective Index of Difficulty $\left(I D_{e}\right.$ ) was measured from $D$ to $w_{e}$, where $D$ is movement distance to the target, and $w_{e}$ is the effective target width. The standard deviation of tapping task that depends on the directions is denoted as $s_{x}$.

ISO recommends that the task has to include many types of difficulty ranges that match the intended use of the input device. There are four precision levels based on the ID (Index of Difficulty), $I D>6$ as high level, $4<I D \leq 6$ as medium level, $3<I D \leq 4$ as low level, and the last, $I D \leq 3$ as very low level. The Index of Difficulty is already defined by the Shannon formulation,

$$
I D=\log _{2}\left(\frac{D}{w}+1\right)
$$

where $D$, similar as previous, is the movement distance parameter, and $w$ is the target width. The $I D$ units are bits.

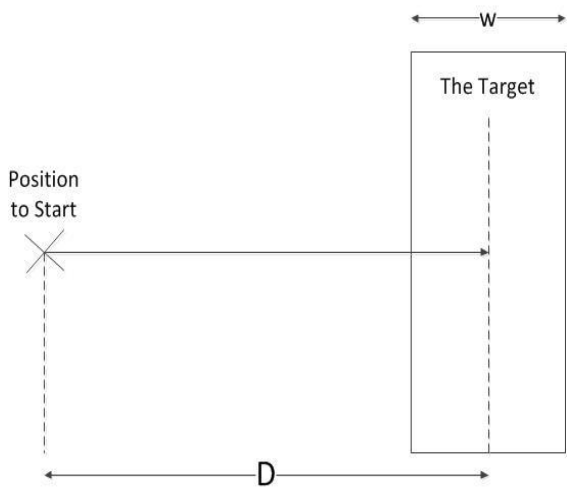

(a)

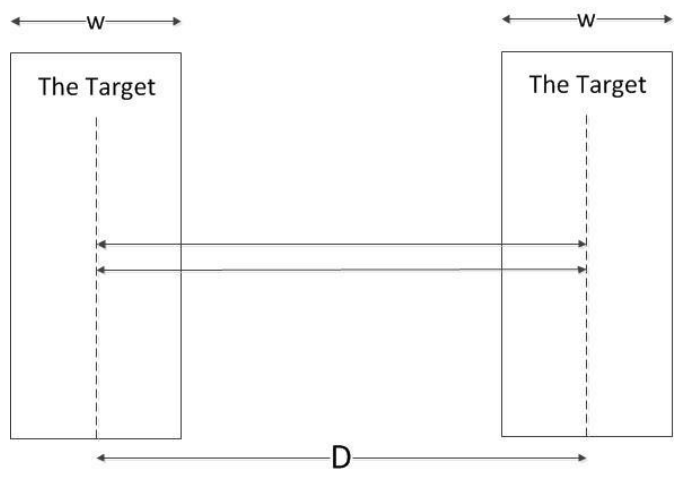

(b)

Fig. 1. One-dimensional tapping task, (a) discrete task, (b) serial task.

The first tapping task model was built by Fitts that used the reciprocal tapping apparatus. The schematic drawing of the tool is described in Figure 1. There are two tasks of Fitts' paradigm, Discrete Task and Serial Task. The assignment for the subjects for Discrete Task was to touch the target plate (without touch the either side of the plate) from the starting position. After the subject finally hit the target, the subject needs to go back to starting point and the task is repeated. The Serial Task has the subjects to hit two targets back and forth continuously between the targets. This paradigm is called one-dimensional, considering the movement only limited for a horizontal line. Either way, whether discrete or serial task, this paradigm is rather obsolete because nowadays movement angle of the pointing device is mostly multi-directional.

Another tapping task is multi-directional tapping task, which is described in Figure 2. The task is tapping a small circle that was begun at the top circle and continues clockwise following the ordered number $[7,8]$. The benefit of this paradigm is that the movement is not restricted to specific area only, but all of the directions [9]. 


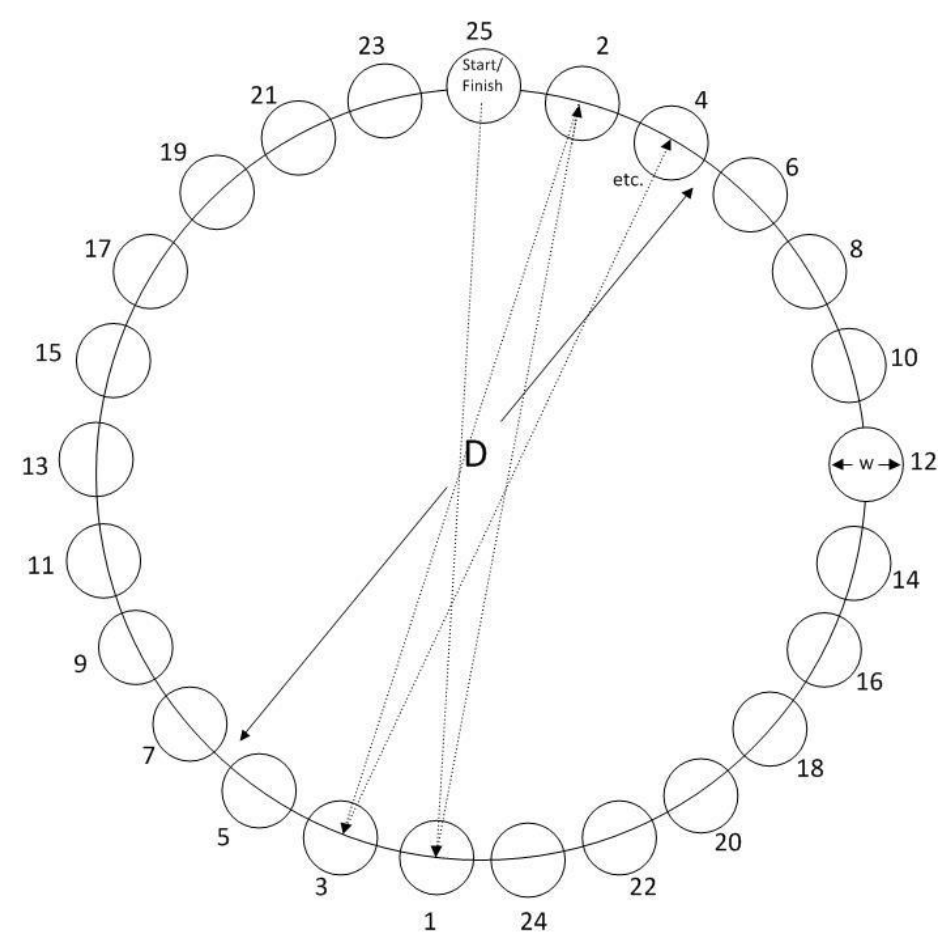

Fig. 2. Multidirectional tapping task.

\subsection{Proof of intersection in very low ID}

In this section, the aim is to prove when the ID is very low or $I D \leq 3$, then the targets circle in the circular arrangement of ISO standard is always intersecting. First of all, the value of $I D \leq 3$ has to be changed into variable $D$ and $w$. Based on Equation 4,

$$
\begin{aligned}
& I D=\log _{2}\left(\frac{D+w}{w}\right) \leq 3 \\
& \Leftrightarrow \frac{D+w}{w} \leq 8 \\
& \Leftrightarrow D+w \leq 8 w \\
& \Leftrightarrow D \leq 7 w
\end{aligned}
$$

The second, the area of overlapping is termed as $A$, see Figure 3; where in the equation to calculate $A$ is already defined by Equation 5 in [10],

$$
A=a^{2} \pi-2 a^{2} \tan ^{-1}\left(\frac{d}{\sqrt{4 a^{2}-d^{2}}}\right)-\frac{1}{2} d \sqrt{4 a^{2}-d^{2}}
$$

with radii of target circle is $a$ and distance between center points of target circles is $d$. Based on the value of ISO standard, the value of $a$ is $\frac{w}{2}$, and the value of $d=0.1253 D$ (this number can be obtained by Pythagorean Theorem), thus Equation 5 becomes 


$$
A=0.7854 w^{2}-\frac{w^{2}}{2} \tan ^{-1}\left(\frac{0.1253 D}{\sqrt{w^{2}-(0.1253 D)^{2}}}\right)-0.0626 D \sqrt{w^{2}-(0.1253 D)^{2}}
$$

The idea of the intersection always exists is the same as the value of (6) is constantly positive. Hence, simply prove that A is consistently positive for $I D \leq 3$.

Theorem 1. For every $D$ and $w$ real positive number that satisfy $D \leq 7 w$ and $w<D$, then $A$ is always real positive.

Proof: For the lower bound, substitute $D=7 w$ to Equation 6, and the value $A=0.0394 w^{2}$ is obtained. For the upper bound, simply remove the subtractions from Equation 6. Consequently,

$$
0.0394 w^{2} \leq A<0.7854 w^{2}
$$

which confirms that the value of $A$ is consistently real positive.

The brute force method also validates that the value of $A$ is always positive, nevertheless it is particularly limited for $D$ and $w$ positive integer numbers.

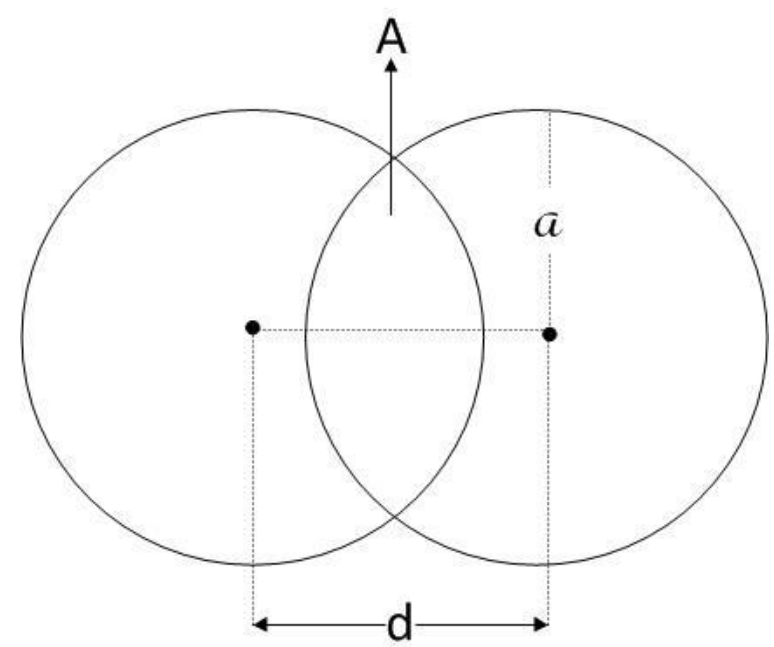

Fig. 3. Description of $A$ as overlapping area, $a$ as radii of target circles, and $d$ as distance of center point between two target circles.

\section{Examples}

Some examples are provided to assist the understanding of the issue. The C\# program has been developed to create the figures. The entire examples were built based on the description in Figure 4 . The space for images in the example of $900 \times 900$ pixels, where the center of the circle is in $(450,450)$ coordinate. 


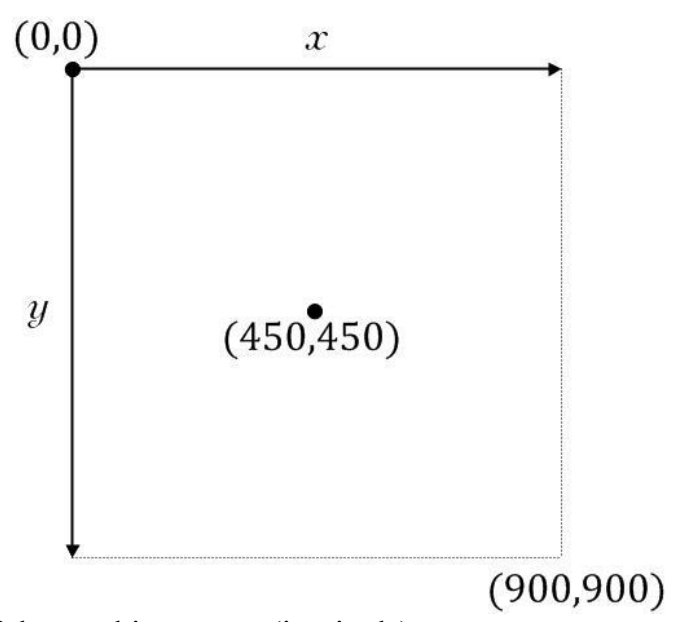

Fig. 4. Description of the working space (in pixels)

All of these cases are very low ID level, or have to comply $D \leq 7 w$; whereas $w<D$. In the following, it is obvious that the intersection invariably occurs.

1. $D=350$ and $w=50$, the result is depicted in Figure 5. Substitute these values to Equation 6 to attain $A=98.6641394$. Based on Equation 6, the boundary of the area is $98.5 \leq A<1963.5$.

2. $D=500$ and $w=80$, the picture is provided in Figure 6 . The value of $\mathrm{A}$ is 586.647212 , where it is bounded by $252.1600 \leq A<5026.5600$.

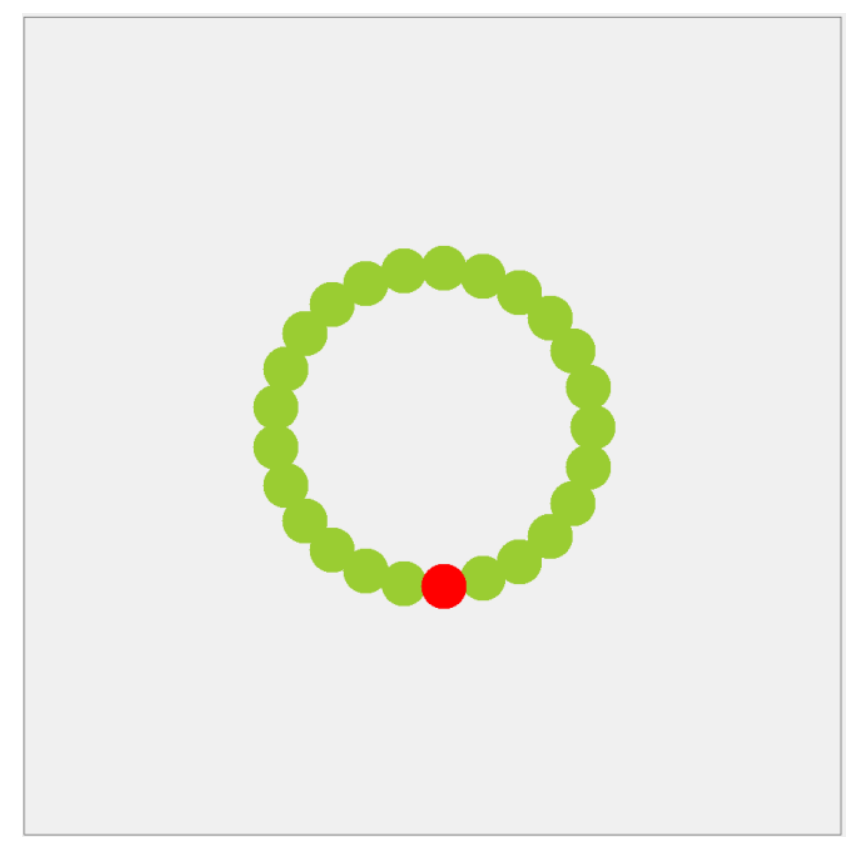

Fig. 5. Multidirectional tapping task for very low $I D$, with $D=350$ and $w=50$. 


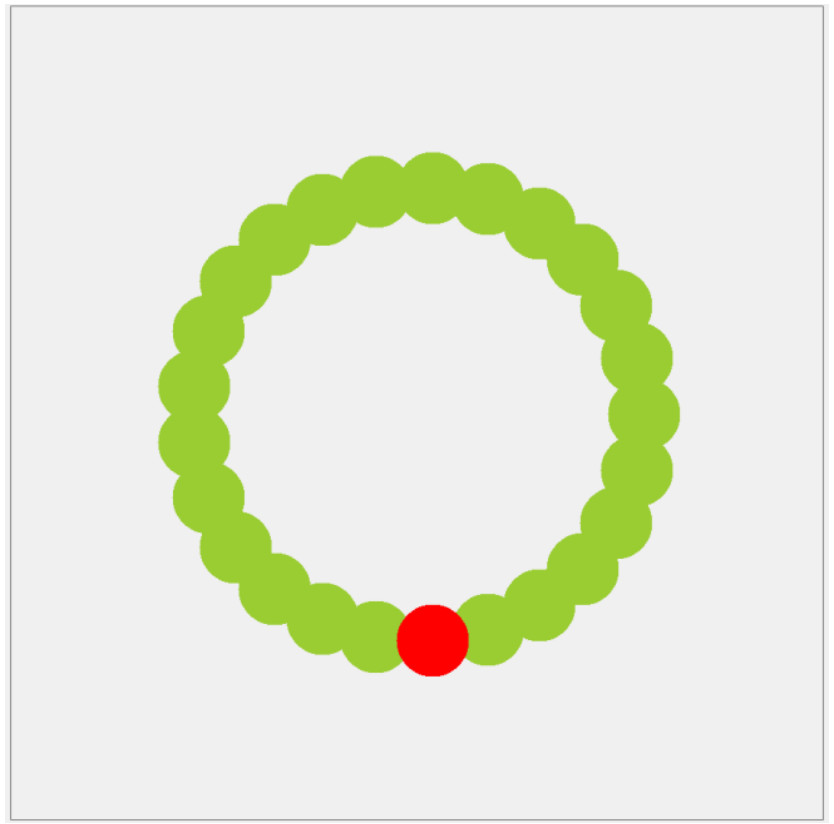

Fig. 6. Multidirectional tapping task for very low $I D$, with $D=500$ and $w=80$.

\section{Conclusion}

This paper is discussing the mathematic proof for overlapping area of multidimensional tapping test in a very low index of difficulty in ISO9241-411. We succeed to prove that when $I D \leq 3$, then the target circles always overlapping with each other. Through this proof, we hope this will be a remarkable condition when design a multi-directional tapping experiment using the ISO standard. The refinement of the effect of overlapping area might be one of the influence conditions in human-computer interaction experiment.

\section{References}

1. K.L. Norman, K.D. Norman. Comparison of relative versus absolute pointing devices [Online] from http://www.cs.umd.edu/hcil/trs/2010-25/2010-25.pdf (2010). [Accessed on 18 December 2018].

2. D. Natapov, S.J. Castellucci, I.S. MacKenzie. ISO 9241-9 evaluation of video game controllers. Graphic Interface 2009 (Kelowna, Canada, 2009). Proceeding of Graphics Interface 2009: 223-230 (2009). pp. 44-45.

https://dl.acm.org/citation.cfm?id=1555930

3. I.S. MacKenzie, T. Kauppinenm, M. Silfverberg. Accuracy measures for evaluating computer pointing devices. Proc. of the ACM SIGCHI Conference on Human Factors in Computing Systems - CHI 2001 (Seattle, USA, 2001). Proceedings of the SIGCHI Conference on Human Factors in Computing Systems:9-16 (2001). https://dl.acm.org/citation.cfm?id=365028

4. J.Y. Oh, W. Stuerzlinger. Laser pointers as collaborative pointing devices. Graphics Interface 2002 (Calgary, Canada, 2002). Proceedings of Graphics Interface 2002:141150 (2002). http://graphicsinterface.org/proceedings/gi2002/gi2002-17/

5. R.B. Widodo, T. Matsumaru. Measuring the Performance of Laser Spot Clicking Techniques. 2013 IEEE International Conference on Robotics and Biomimetics (ROBIO) (Shenzhen, China, 2013). http://ieeexplore.ieee.org/document/6739639/ 
6. I.S. MacKenzie. Human-Computer Interaction: An Empirical Research Perspective. Waltham: Elsevier Inc (2013). pp. 74-86.

https://books.google.co.id/books?id=k0kBgyCaokAC\&printsec=frontcover\&dq $=$ Huma n-

Computer+Interaction:+An+Empirical+Research+Perspective.+Waltham:+Elsevier+In c\&hl=en\&sa=X\&ved=0ahUKEwjBo_L6qcHZAhVFvo8KHY6sC_QQ6AEIKDAA\#v= onepage\&q=Human-

Computer $\% 20$ Interaction $\% 3 \mathrm{~A} \% 20 \mathrm{An} \% 20$ Empirical $\% 20$ Research $\% 20$ Perspective. $\% 2$ 0Waltham $\% 3 \mathrm{~A} \% 20$ Elsevier $\% 20$ Inc \&f $\mathrm{f}=$ false

7. ISO. Reference number ISO/TS 9241-411:2012(E). International Organization for Standardization (2012). https://www.iso.org/standard/54106.html

8. I.S. MacKenzie. Fitts'law. In: Handbook of human-computer interaction. K.L. Norman, J. Kirakowski (Eds.). Wiley: Hoboken, NJ (2018) pp. 349-370. https://www.wiley.com/enus/The+Wiley + Handbook + of + Human + Computer+Interaction + Set-p-9781118977279

9. R.W. Soukoreff, I.S. MacKenzie. Int. Journal of Human-Computer Studies, 61:751789 (2004). http://www.yorku.ca/mack/ijhcs2004.pdf

10. F.W. Weisstein. I.ens [Onlinel from http://mathworld.wolfram.com/Lens.html (2007). [Accesed on 18 December 2017]. 